\title{
Le fonds sigillographique de la BNU, une collection méconnue
}

Daniel Bornemann

\section{(2) OpenEdition}

12 Journals

Édition électronique

URL : http://journals.openedition.org/rbnu/601

DOI : $10.4000 /$ rbnu. 601

ISSN : 2679-6104

Éditeur

Bibliothèque nationale et universitaire de Strasbourg

Édition imprimée

Date de publication : 1 novembre 2018

Pagination : 34-43

ISBN : 9782859230784

ISSN : 2109-2761

\section{Référence électronique}

Daniel Bornemann, « Le fonds sigillographique de la BNU, une collection méconnue », La Revue de la BNU [En ligne], 18 | 2018, mis en ligne le 01 juillet 2019, consulté le 15 décembre 2020. URL : http:// journals.openedition.org/rbnu/601 ; DOI : https://doi.org/10.4000/rbnu.601

\section{(c) (i) (8)}

La Revue de la BNU est mise à disposition selon les termes de la Licence Creative Commons Attribution - Pas d'Utilisation Commerciale - Partage dans les Mêmes Conditions 4.0 International. 


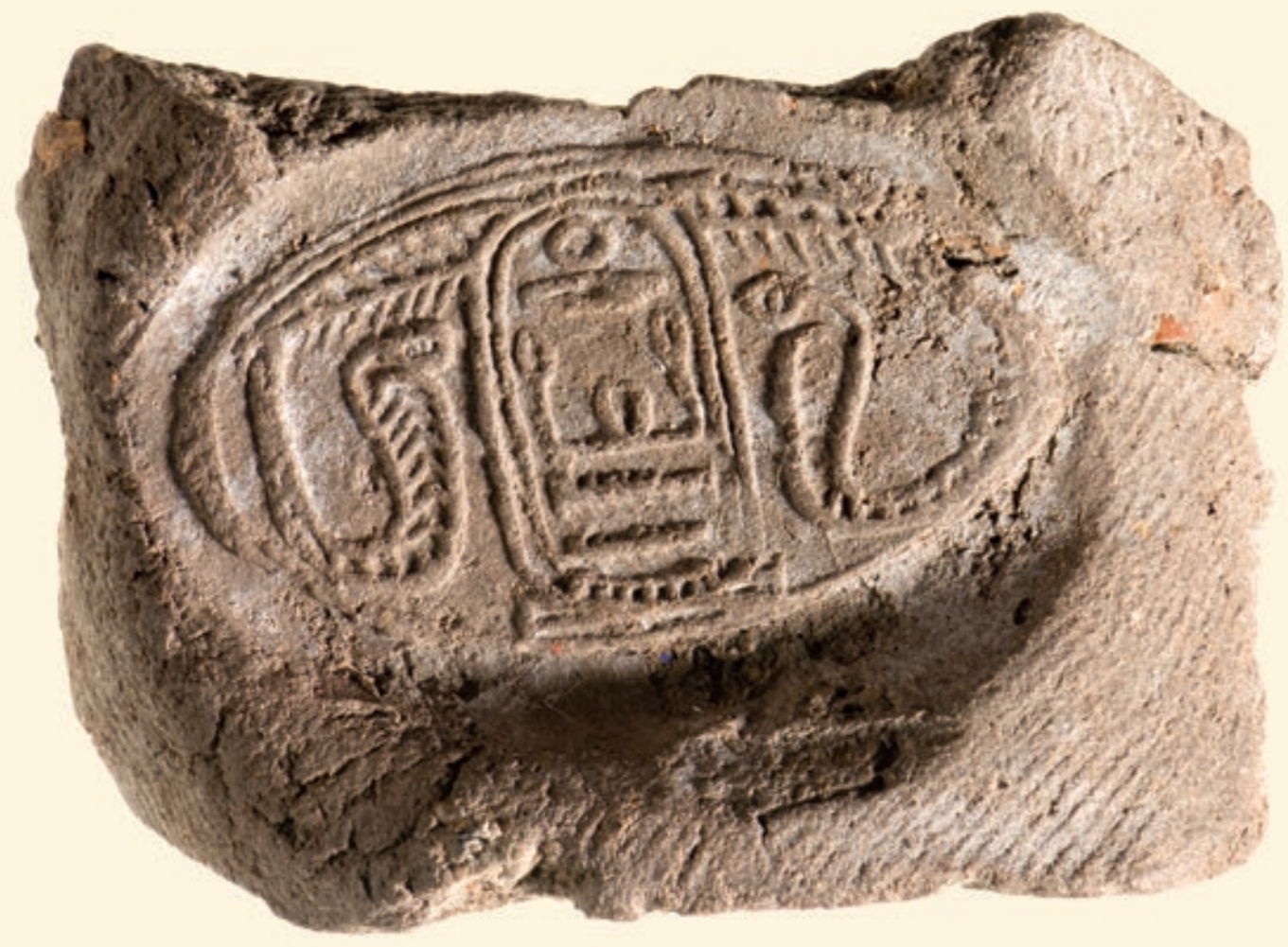




\title{
LE FONDS SIGILLOGRAPHIQUE DE LA BNU, UNE COLLECTION MÉCONNUE
}

\author{
PAR DANIEL BORNEMANN
}

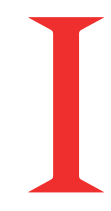

1 y a un an paraissait l'ouvrage de Daniel Keller : Le sceau, empreinte de l'Histoire : sigillographes et sigillographie en Alsace ${ }^{1}$, un manuel de sigillographie générale et locale qui fait aussi le tour des collections de notre région. La BNU y est mentionnée à une place modeste avec une description sommaire de ses ressources. En Alsace, bien des institutions sont plus riches en sceaux. Pensons aux services d'archives départementales ou municipales ayant conservé leurs chartes et pièces anciennes; pensons bien entendu au Musée du Sceau à La Petite Pierre. À la BNU, numériquement, nous ne saurions rivaliser avec ces institutions. Cependant notre collection sigillographique possède deux spécificités qui en font un lieu important pour cette science : la présence de sceaux provenant d'autres horizons et d'autres espaces chronologiques d'une part (sceaux antiques, cylindriques mésopotamiens, égyptiens, persans, orientaux, sur une multiplicité de supports particuliers et rares), et d'autre part une collection de copies d'empreintes de sceaux alsaciens, lorrains, rhénans et allemands qui vise l'exhaustivité et qui, parce qu'elle a son histoire, mérite d'être connue du public.

Le sceau est un objet archéologique qui a la particularité d'être souvent assujetti à un document daté et localisé, ce qui fait de lui un témoin privilégié dans bien des contextes historiques. Cela fait dire au comte de Laborde : "Quand on réfléchit que chacun de ces monuments iconographiques, fait par ordre de celui qu'il représente, et pour ainsi dire sous ses yeux, de manière à traduire sa personne au gré de ses caprices, a été attaché de ses mains à un document dicté par lui, qui nous instruit, à la date indiquée, de ses noms et qualités, du lieu de sa résidence et de ses intérêts les plus chers, il faut convenir que la sigillographie n'est ni une science vaine, ni une occupation stérile, qu'elle est au contraire la plus autorisée des branches de l'archéologie $"{ }^{2}$. Il est question ici d'archéologie médiévale occidentale, mais on peut étendre sa remarque à l'Antiquité, au moins dans une certaine mesure. En effet, dès l'âge du papyrus comme à celui de l'argile mésopotamienne, des empreintes de sceaux sont associées à des textes divers et permettent donc de faire un lien entre un fait et son contexte.

La BNU possède donc un corpus de sigillographie antique conséquent, dont nous tenterons de donner un bref aperçu. En premier lieu, citons les sceaux cylindriques mésopotamiens, qui sont au nombre de 15. De taille variable, ciselés dans des minéraux divers (mais toujours dans la pierre dure), certains d'entre eux ont pu être déchiffrés et édités par Karl Frank ${ }^{3}$ en 1928. Ce petit fonds se double d'une importante série de documents en écriture cunéiforme (tablettes d'argile), comportant des empreintes de sceaux imprimées dans le support encore meuble, il y a 40 ou 50 siècles. Cet ensemble est du plus haut intérêt pour l'archéologie orientale. En effet, la valeur du document s'en trouve 
pour ainsi dire « mise au carré », puisque non seulement l'inscription et le motif iconographique du sceau ont pu se conserver, mais en plus ces éléments sont associés à une information documentaire circonstanciée. L'un ne s'ajoute pas seulement à l'autre mais interagit, insuffle potentiellement de nouvelles connaissances dans le monde des spécialistes. Le sceau est un outil unique qui permet des marquages multiples, et dans l'idéal il peut être un trait d'union entre plusieurs documents et former ainsi des "dossiers " réunissant des objets différents sous un dénominateur commun. Il faut pourtant faire preuve d'optimisme, car dans la réalité il existe un grand nombre de sceaux cylindriques retrouvés dans des fouilles et aujourd'hui édités, mais peu d'occurrences d'empreintes identiques relevées sur des tablettes cunéiformes. Peut-être en existe-t-il, mais les liens n'ont pas encore été faits.

Les corpus de sceaux cylindriques sont édités depuis longtemps, de deux façons, en deux ou en trois dimensions : ils sont reproduits soit par des gravures, photographies ou dessins, soit par des empreintes diffusées par copies et moulages. Au $19^{\mathrm{e}}$ siècle déjà, l'étude des sceaux antiques (hittites, babyloniens, sumériens, akkadiens, sassanides, etc.) a suscité des échanges de moulages entre institutions et spécialistes. La BNU conserve ainsi quelques empreintes modernes ${ }^{4}$ de sceaux utilisés dans l'Antiquité, soigneusement réalisées par des institutions comme le Metropolitan Museum de New York, ou encore les collections impériales de Vienne et de Berlin.

Les collections de la BNU comptent par ailleurs deux matrices de sceaux égyptiens qui ont la forme de scarabées en ivoire ou en terre cuite vernissée. La collection de papyrus est également intéressante du point de vue de la sigillographie. Certains contrats sur papyrus, exactement comme des chartes médiévales, nécessitaient l'utilisation de sceaux. Ceux-ci n'étaient que des boulettes de terre meuble sur lesquelles on pressait une intaille ou le chaton d'une bague. Parfois on reconnaît encore quelques signes hiéroglyphiques dans un cartouche ; souvent l'écriture semble avoir disparu pour ne laisser subsister que le motif grécolatin d'un beau profil ou d'un symbole mythologique. La BNU ne conserve pas d'intaille (sinon moderne), mais possède quelques empreintes de sceaux attachées à des documents. Là aussi, l'un peut éclairer l'autre et former un contexte. Mais en réalité ces sceaux parlent peu et sont souvent très abîmés, lorsqu'ils ne sont pas tout simplement détachés du contrat qu'ils accompagnaient. Heureusement, certains sceaux ont été conservés attachés à leur papyrus d'origine, dans un dispositif garantissant leur conservation.

À la charnière entre l'Antiquité et le MoyenÂge on trouve les bulles papales, type particulier de sceaux en plomb. Mais l'empreinte d'un sceau peut se faire dans toute matière meuble : terre, cire, métal ou verre en fusion. La BNU possède ainsi une collection d'estampilles et de poids monétaires en verre, porteurs d'écriture arabe. Cette collection comporte des fragments de scellés de verre, qui étaient apposés sur la fermeture de contenants afin de garantir le contenu ou le volume. L'estampille était imprimée dans le verre encore mou qui servait à «luter» l'ouverture. Quoiqu'indirectement, cette collection peut s'apparenter au vaste domaine de la sigillographie. Le monde islamique est également présent dans notre collection par des témoins variés : moulages d'empreintes de sceaux de personnages de haut rang, les «tugras », ou encore entités qu'on pourrait nommer des combinaisons de personnages et d'outils sigillographiques. Deux orientalistes présents pour ainsi dire en personne dans les collections de la BNU, Arthur de Gobineau et Julius Euting, ont en effet enrichi la bibliothèque, après leur mort, d'exemples de ce lien entre un individu et des sceaux. Il s'agit dans leurs cas d'intailles, ces incisions figuratives ou écrites faites dans des pierres dures, montées ensuite sur des bagues ou des supports spéciaux, qui étaient encore en usage à la fin $\mathrm{du} 19^{\mathrm{e}}$ siècle en Orient. Gobineau a ainsi rapporté de ses voyages en Perse trois intailles montées sur des supports de métal, ainsi qu'une bague porte-sceau. Euting, qui voyagea en Arabie et dans le Proche- Orient, a également utilisé deux bagues, l'une en or, l'autre en argent, dont les chatons portent, en creux ou en "relief sortant", des inscriptions. Ces objets servaient réellement à nos voyageurs, il y a un siècle et demi, de signature, de sceau, de témoignage de leur propre authenticité.

Ces éléments de sigillographie ancienne et orientale sont sans doute ce par quoi notre collection trouve sa plus grande originalité. Mais la haute époque de la sigillographie occidentale y occupe également une place de choix. Ce qui paraît plus original, et qui ne se trouve qu'à la $\mathrm{BNU}^{5}$, c'est la collection de moulages 

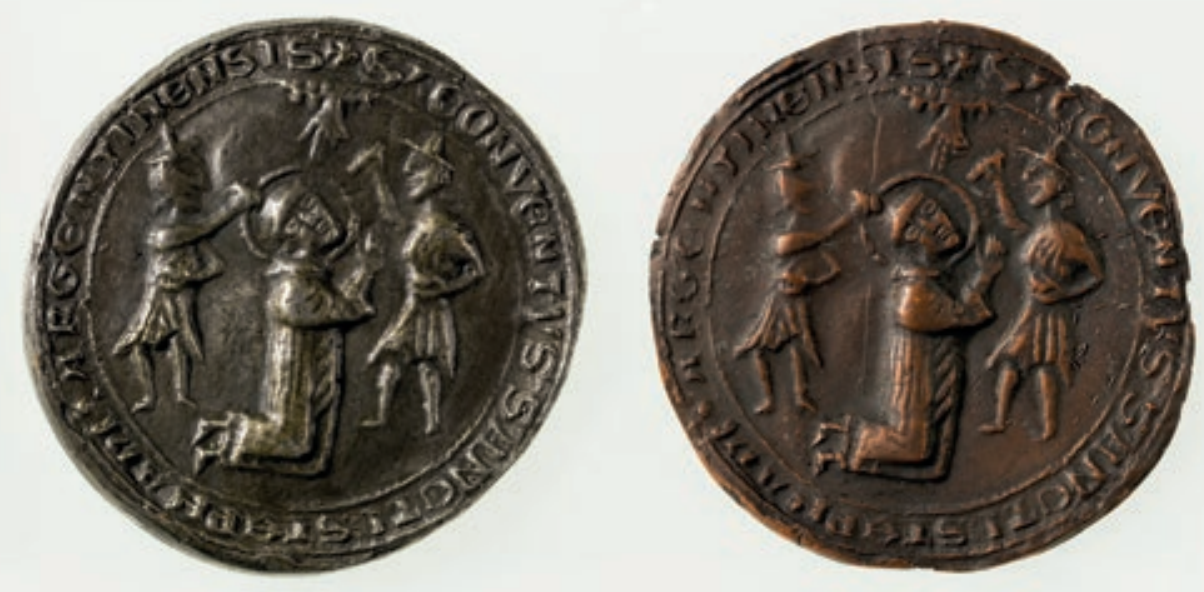

S. conventus : Sancti : Stephani : Argentinensis. Sceau du couvent de Saint-Etienne, de 1264. Un original est conservé aux Archives municipales de Strasbourg, sous la cote AAu.1395. Deux moulages en alliages métalliques différents ; celui de gauche a été fabriqué selon la méthode de Röckl (diamètre $55 \mathrm{~mm}$; coll. BNU).

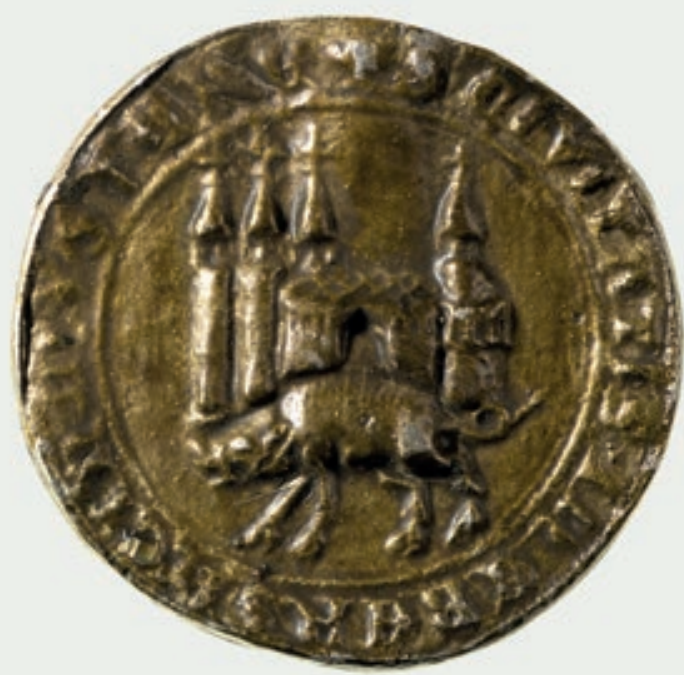

S. civitatis. di. Ebersheim Munstere. Sceau de la cité d'Ebersheim Münster (ou Ebersmunster). Moulage en alliage métallique.

Conforme à l'héraldique, ce sceau utilisé en 1262 ou 1264

porte une représentation de l'église remplacée par l'actuelle abbatiale

au $18^{\mathrm{e}}$ siècle. Un original est conservé aux Archives municipales de Strasbourg, sous la cote AAu.1393 (diamètre $44 \mathrm{~mm}$; coll. BNU). 
de sceaux alsaciens, lorrains et germaniques ${ }^{6}$ qui a été constituée à partir de 1879 par les conservateurs de la bibliothèque, sous le contrôle du ministère des Cultes et de l'Enseignement d'Alsace-Lorraine, en liaison avec toutes les institutions susceptibles de conserver des sceaux dans le Reichsland et au-delà.

La sigillographie est en effet une science qui s'est éveillée au milieu du $19^{\mathrm{e}}$ siècle. Les auteurs des principaux manuels insistent sur cette soudaine et tardive naissance. Dès son origine, la fragilité des sceaux et les difficultés de leur communication, les risques pris à chaque manipulation, ont amené les archivistes à envisager la création de collections de copies en relief qui permettaient elles-mêmes d'en tirer d'autres copies. En France ${ }^{7}$, c'est en 1832 que les premiers pas dans cette direction sont faits. Entre 1842 et 1850, la pratique prend un essor national. Les principaux dépôts parisiens sont exploités, et l'opération s'étend à partir de la capitale, englobant quelques régions au nord-ouest, puis au sud-est à partir de 1861. En 1863, on atteint le chiffre de 11840 moulages réalisés. Et c'est à ce moment qu'intervient la guerre de 1870 : l'Alsace n'a pas été explorée par les équipes parisiennes, et ne le sera plus avant très longtemps. Or durant le temps de l'annexion, le quadrillage de la France se poursuit pour atteindre en 1905 le chiffre de 54709 moulages réalisés. L'opération est d'envergure et la France bien couverte, mais l'Alsace est passée entre les mailles du filet, en raison de sa destinée particulière. Une espèce de vide bibliographique s'installe : les ouvrages généraux ne mentionnent pas l'Alsace, et on peut parcourir des traités entiers sans rencontrer un mot sur notre région. La Moselle, avec Metz en particulier, quoiqu'elle ait partagé le sort de l'Alsace pour ce qui est de la campagne de moulages, est toutefois bien représentée, de façon plus générale, dans les études françaises. On se doute que plusieurs facteurs ont joué pour créer ce phénomène «géo-culturel» particulier (l'Alsace ressortissant globalement plus de l'aire culturelle germanique). Mais revenons à la collection de moulages de la BNU.

Celle-ci est donc constituée à partir des sceaux provenant des institutions du Reichsland. Les procédés mis en œuvre sont divers : copies en plâtre, empreintes originales, et un grand nombre de reproductions en métal. La création de cette collection s'accompagne d'une réflexion sur la méthodologie et les techniques utilisées. Un premier procédé est le moulage et la réalisation d'une copie en plâtre, support qui peut être colorié. Une expertise sur l'utilisation du plâtre est ainsi menée par le professeur Johannes Thiele (1865-1917) à l'Institut de chimie de l'Université de Strasbourg en 1910-1912. Un autre procédé, appelé « Röckl'sche Methode ", la méthode du chimiste allemand Max Albert Röck $1^{8}$, consistant en la réalisation de moulages en métal souple sur un moule en plâtre, a lui aussi été utilisé. Enfin, il y a le procédé de François Fastinger (1842-1916) ${ }^{9}$, secrétaire des archives départementales du Bas-Rhin entre 1889 et 1905, qui consiste à prendre une empreinte en creux du sceau, à l'enduire de graphite, puis à fabriquer une empreinte en relief en y coulant un alliage à base d'étain. Plusieurs alliages différents sont utilisés, aux reflets plombés, ferreux, cuivrés ou noirâtres. Fastinger, dont la méthode est une amélioration de celle de Röckl, prétend faire reconnaître la supériorité de son procédé et en détaille les avantages dans une lettre aux conservateurs de la bibliothèque.

L'archiviste Ernest Hauviller ${ }^{10}$, des archives départementales du Haut-Rhin et de la Moselle ( « Bezirksarchiv » de Colmar et de Metz) a lui aussi joué un rôle important dans la constitution de collections de moulages, et peut être cité comme " passeur " de méthodes sigillographiques entre France et Allemagne. Dans ce dernier pays, il n'y avait aucune collection sigillographique centrale ; on avait affaire à une fédération d'États. Seules des initiatives privées ou locales avaient été lancées. La plus remarquable était la collection de Munich, où Röckl avait œuvré. L'appel d'Hauviller pour une prise en charge nationale de la question sigillographique est resté vain, puisqu'Erich Kittel, plus d'un demi-siècle après, a tenté de le relancer ${ }^{11}$. Ce qui restait à faire dans la plupart des Länder allemands au milieu du $20^{\mathrm{e}}$ siècle avait été réussi en Alsace-Moselle. Mais là encore, et on comprend pourquoi, la collection strasbourgeoise est restée ignorée par le répertoire des collections de moulages de sceaux allemandes qu'a dressé Kittel. La collection de l'actuelle BNU est donc passée dans un angle mort : ni les répertoires français, ni ceux concernant l'Allemagne, n'en ont fait mention.

Rentrons maintenant dans quelques détails de cet ensemble de moulages. La campagne de recueil de copies est orchestrée à partir du ministère des Cultes 


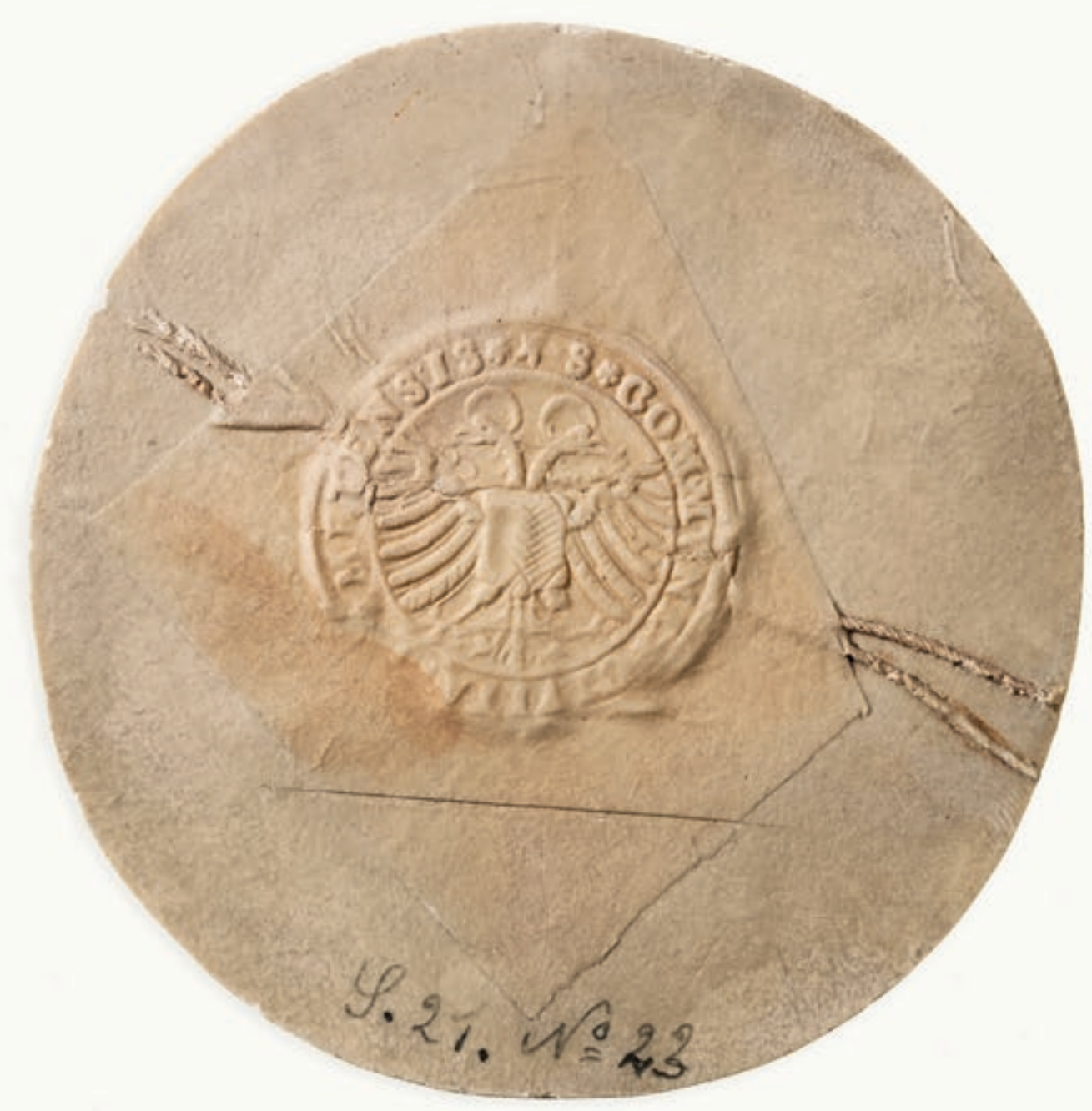

S[igillum] commune civitatis Metensis. Sceau de la ville de Metz, 1623.

Moulage en plâtre du sceau sous papier. L'original est conservé aux Archives départementales de la Moselle, série 21, $\mathrm{n}^{\circ} 23$. Diamètre du sceau : $37 \mathrm{~mm}$. Le rendu du moulage est remarquable (coll. BNU). 
et de l'Enseignement, directement par le secrétaire d'État (Staatssekretär). Les sceaux sont réalisés par un atelier situé auprès du ministère, puisque des courriers mentionnent des prêts de chartes à destination de cette administration, et l'on veille avec scrupule à tous les détails, jusqu'à la gestion de la caisse de transport. Une fois réalisés en deux exemplaires, les moulages sont expédiés à la bibliothèque, où les trois administrateurs successifs, Karl August Barack, Julius Euting et Georg Wolfram les réceptionnent. Le professeur Ludwig Müller, en charge du cabinet numismatique du Reichsland (la "Landesmünzsammlung "), les inventorie ${ }^{12}$ et établit leur description. L'un des exemplaires positifs est expédié à l'institution qui possède l'original. L'autre exemplaire positif est gardé par la Landesmünzsammlung. L'empreinte du sceau en négatif est visiblement gardée par le ministère, ou alors elle est gardée par l'atelier qui l'a réalisée. À ce sujet, il faut signaler le cas de Fastinger, qui a pu vendre pour son propre compte des moulages de sceaux au ministère, et donc à la bibliothèque. Lors d'une exposition de moulages de sceaux à la KULB ${ }^{13}$, en 1910, il va jusqu'à demander que son droit d'auteur soit reconnu sur ces copies. C'est donc qu'il les réalisait en marge de ses activités professionnelles, à titre privé ${ }^{14}$. Toutes les grandes institutions sont contactées et leurs responsables coopèrent activement. Les coûts sont pris en compte par la Caisse centrale du Reichsland, qui réclame des justificatifs, et la Cour des comptes inspecte le tout. Cette activité était alors dotée d'un budget de 200 à 300 marks par an, la collection étant composée de pièces achetées à des institutions ou à des particuliers. La Société lorraine d'histoire et d'archéologie (Gesellschaft für lothringische Geschichte und Altertumskunde) de Metz a édité vers 1910 un catalogue des reproductions de sceaux qu'elle proposait à la vente, en précisant les prix dans une seconde édition en 1911. Ce service messin a même servi d'intermédiaire entre Strasbourg et Paris pour la fourniture de moulages fabriqués par les Archives nationales, à partir de pièces lorraines conservées à Paris.

Cette collection, où très peu de sceaux apparaissent en double (trois sceaux sont représentés par deux empreintes ou plus), compte 849 empreintes. Elles sont en grande majorité en métal. La collection compte aussi 9 empreintes originales de sceaux, malheureuse- ment séparées de leurs pièces d'origine, et encore présentées dans leurs capsules en bois tourné. Enfin, une très courte série de 21 matrices anciennes et modernes, accompagnées de plusieurs empreintes, vient compléter le tout. Curieusement, les matrices sont moins parlantes que les empreintes. En effet, comme nous l'avons souligné au début de cet article, le sceau devient un point d'appui pour l'Histoire à partir du moment où il permet de relier entre eux un personnage, un lieu, une date et un acte. La matrice seule, ou accompagnée de sa seule empreinte, ne donne qu'un nom, parfois un prénom, avec éventuellement des abréviations et une fonction, mais rien de plus : ni lieu, ni sujet, ni date. Ce n'est qu'une signature sans objet ni contexte.

Le budget alloué à la sigillographie pouvait éventuellement servir aussi à l'achat de collections entières de sceaux. Quelques acquisitions furent envisagées. Julius Euting refuse ainsi l'achat d'une collection privée strasbourgeoise en 1907, jugeant que celle-ci manquait de cohérence et n'avait pas de caractère alsatique. Plus tard, en février-avril 1917, un achat important est effectué : celui de la collection de 6000 empreintes de sceaux en cire d'Espagne réunie par le Dr. Stach von Goltzheim, de Dieuze en Moselle (appelée «Duss » dans les échanges de courriers). Cette collection est composée d'empreintes de sceaux en cire, de petite taille, utilisés pour cacheter les courriers envoyés par la poste aux $18^{\mathrm{e}}$ et $19^{\mathrm{e}}$ siècles. La chronologie est plutôt récente, mais l'essentiel du « Gotha » y est représenté. Les empreintes de sceaux en cire rouge, noire ou verte, parfois même bicolore, sont classées selon l'initiale de la famille concernée. La fin de la Première Guerre mondiale eut pour conséquence que cet achat fut imputé au budget général de la Bibliothèque universitaire et régionale de Strasbourg ${ }^{15}$ redevenue française.

Durant cette même Première Guerre mondiale, d'autres objets d'intérêt sigillographique ont intégré la BNU. Ainsi la cour supérieure impériale du Reichsland (Kaiserliches Oberlandesgericht), à Colmar, a livré à la bibliothèque 282 cachets en cuivre et 196 en fer, provenant des anciennes juridictions alsaciennes et lorraines datant d'époques révolues, ce qui représente tout de même 478 pièces. Ce dépôt est daté de fin mars 1916. Il s'agit de matrices de cachets à encrer, où les caractères sont en relief sortant, et de matrices de sceaux où les caractères sont en creux. Comment interpréter 

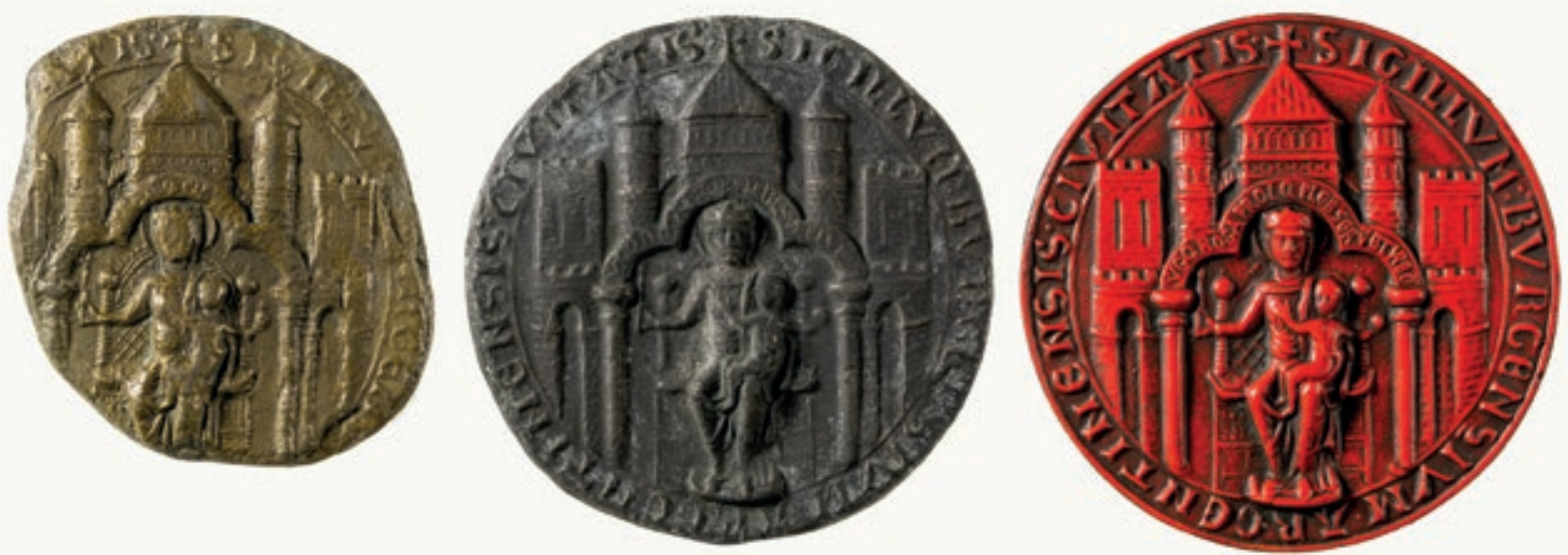

Sigillum burgensium Argentinensis civitatis + Virgo roga prol[em] q[uod] pleb[em] ser[vet]

et urb[em]. Trois moulages du grand sceau de Strasbourg, le plus ancien connu. À gauche, moulage en alliage de métal sur un original daté de 1201 ; au milieu, sur un original daté de 1295 ; à droite par M. Haudot, reconstitué d'après un original de 1285 (diamètre du sceau complet : $97 \mathrm{~mm}$; coll. BNU).

ce geste : mise à l'abri dans une collection publique de ces outils afin qu'ils ne soient pas mal utilisés, ou réflexe de protection d'un patrimoine sigillographique administratif en danger?

Depuis cette période, des sceaux sont entrés dans les collections par différents canaux : achats, dons et dépôts ${ }^{16}$ sont susceptibles de contenir des originaux de sceaux (empreintes liées à un document), soit appendus à des chartes ou documents de cet ordre, soit appartenant au domaine des cachets utilisés dans les correspondances. Celles datant de la charnière des $18^{\mathrm{e}}$ et $19^{\mathrm{e}}$ siècles sont particulièrement riches en cachets de cire, quand les archives administratives le sont en sceaux sous papier et en cachets, souvent très élaborés. Toute bibliothèque ou tout fonds d'archives possède de tels originaux, en plus ou moins grande quantité, et la BNU n'a là-dessus aucune originalité à signaler. Du point de vue des collections de sceaux, les Archives municipales de Strasbourg, les archives départementales des Bas-Rhin, Haut-Rhin et Moselle, ou encore la Bibliothèque Humaniste de Sélestat ont de très grandes richesses à mettre en avant. La BNU, quant à elle, se concentre sur la restauration et le conditionnement des chartes et documents portant des sceaux, où son atelier a acquis une grande maîtrise.

Une dernière grande époque peut cependant être signalée : celle où Charles Haudot (1919-2010) a réalisé un très grand nombre de moulages de sceaux en silicone. Sigillographe de la Ville de Strasbourg, il a contribué à la fondation du Musée du Sceau déjà cité, qui présente un grand nombre (environ 2500 ) de fac-similés d'empreintes se rapportant à l'histoire de la région. Il a réalisé lui-même les empreintes en utilisant des moulages en creux confectionnés des années plus tôt par Fastinger. Il a édité, ou réédité en les révisant, les données de la sigillographie alsacienne ${ }^{17}$. Sauf exception, il n'a pas eu besoin de recourir à la collection de moulages de la BNU, mais il a confié à 


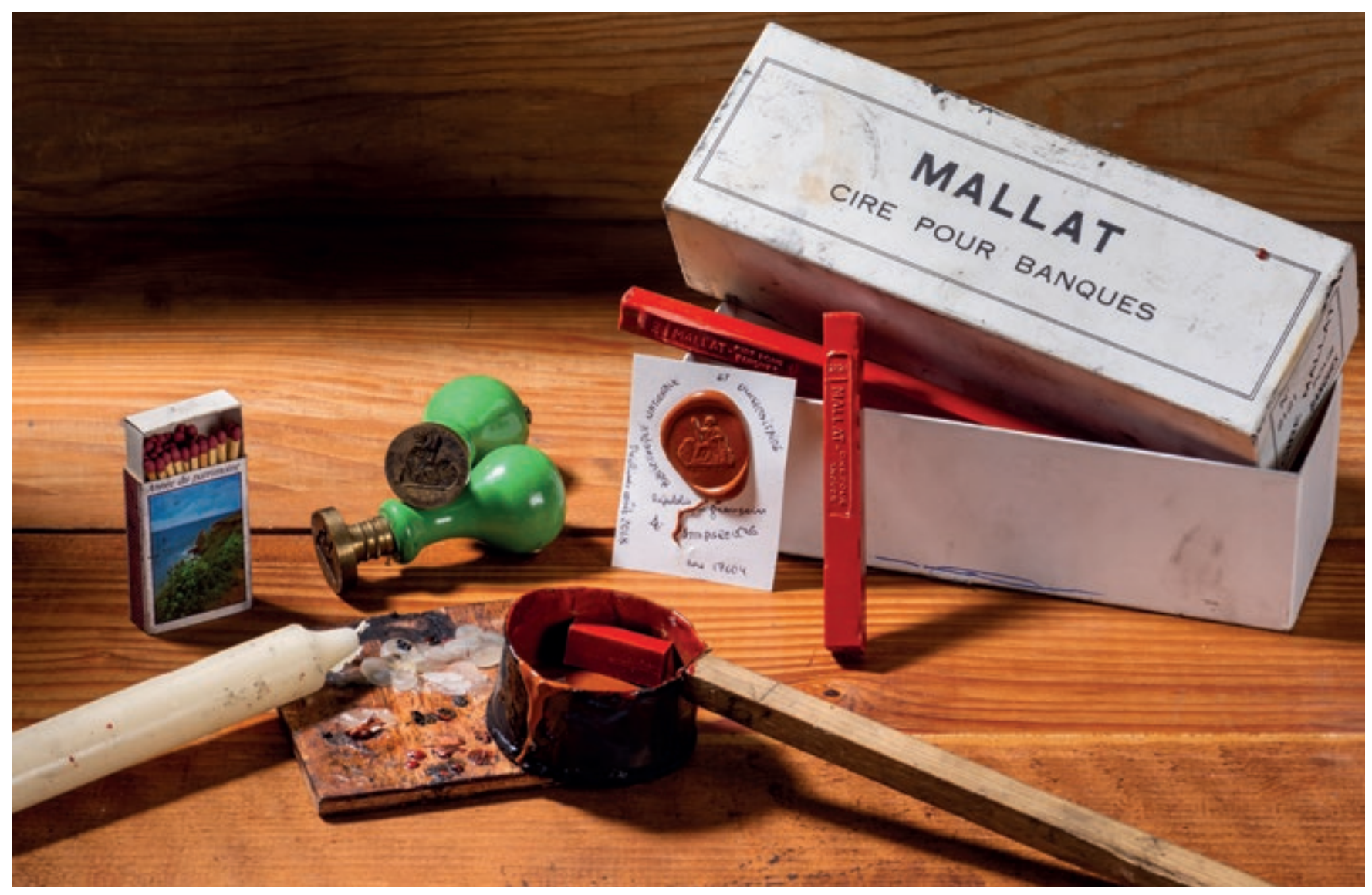

Le sceau de la BNU : l'empreinte, la matrice (2 exemplaires) et le matériel nécessaire à sa réalisation (coll. BNU)

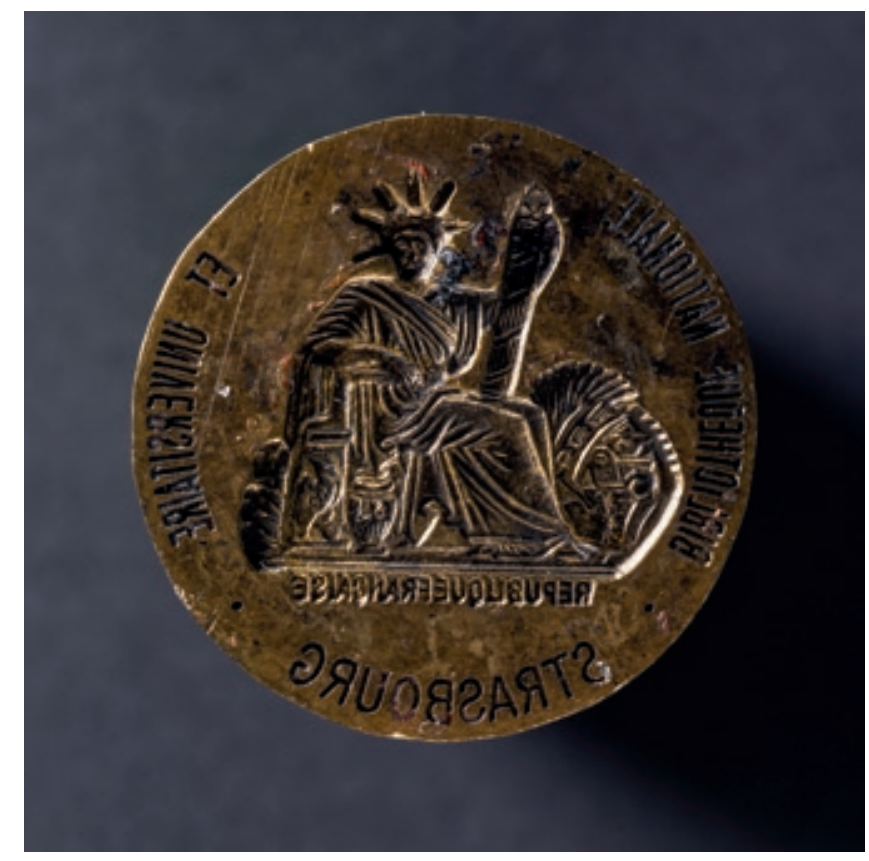

Le sceau de la BNU : matrice (coll. BNU) 
la bibliothèque de belles réalisations : certains moulages en résine synthétique sont ainsi venus combler des lacunes de la collection. Ces réalisations récentes peuvent surprendre par leur aspect. Leurs couleurs vives, leur aspect tout neuf étonnent au milieu d'un ensemble tout de même très patiné. C'est que nous sommes habitués à des sceaux qui ont subi les affronts du très grand âge et qui ont pris et incorporé la poussière. Le propos de Charles Haudot était, semble-t-il, de restituer dans leur état natif ces empreintes, et de gommer leurs siècles d'avanies. Ces pièces réalisées en silicone ou en caoutchouc synthétique ont donc l'intérêt supplémentaire de permettre une vision absolue de l'empreinte du sceau, telle qu'elle pouvait se présenter au moment de sa réalisation. C'est donc un retour vers le passé, et la perception d'une esthétique particulière qu'elles permettent, avec l'effet de saisissement que cela peut provoquer.

La sigillographie est une affaire de reliefs, de creux et de bosses, autrement dit de « 3D ». Les techniques de moulage ont permis un accès facilité, et sans danger pour eux, aux sceaux, témoins fragiles des anciens temps. À l'âge informatique, l'outil numérique facilite la transmission de l'information et la mise à disposition libre et gratuite des biens culturels, en images, en sons, et même en relief. La collection de moulages de sceaux de la BNU pourrait faire l'objet d'une numérisation en 3D. En effet, la variété des objets conservés, allant de la protohistoire jusqu'au 19e, voire au $20^{\mathrm{e}}$ siècle, la variété des matériaux, des types ou des origines, justifieraient une opération de mise en valeur particulière. La combinaison de l'ensemble pourrait de plus être motivante pour d'autres institutions conservant ce type de documents. Rappelons que la diffusion de la sigillographie repose jusqu'à nos jours sur la photographie (voire le dessin ou la gravure), donc sur un mode en deux dimensions. Aussi efficace que puisse être la meilleure des photos, elle ne permet pas de tout distinguer car le jeu de l'éclairage, comme en épigraphie ou en numismatique, crée des difficultés. De plus, les moulages avaient l'avantage de conserver à l'objet sa taille d'origine, ce qui n'est pas à négliger. Faudra-t-il donc imaginer une transposition systématique en $3 \mathrm{D}^{18}$ ?
La BNU possède elle-même son propre sceau (voir ill. ci-contre). Celui-ci a pu servir dans certaines démarches administratives, comme des concours où des enveloppes doivent être scellées. Grâce au matériel de fabrication qui l'accompagne, elle pourra en cas de besoin recourir encore à la pratique sigillographique.

\section{NOTES}

1- Fédération des sociétés d'histoire et d'archéologie d'Alsace, collection « Alsace-Histoire », tome 10, 2017

2- Laborde, Léon de, La collection des empreintes de sceaux des archives de l'Empire et son inventaire, Paris, Renouard, 1863, p. 8

3- Strassburger Keilschrifttexte in sumerischer und babylonischer Sprache, Berlin, De Gruyter, 1928

4- Sous le numéro d'inventaire INV. 2014.1.2

5- Plusieurs services d'archives possèdent des collections plus ou moins étendues, mais qui ne sont pas comparables avec celle conservée à la BNU.

6- Les dossiers d'archives relatant la constitution de cette collection ont pour cotes AL.51,140 et AL.51,140 bis.

7- Voir pour cela le chapitre «II : Nécessité des collections de moulages » dans l'ouvrage d'Auguste Coulon, Le service sigillographique des Archives nationales, Paris, 1916, et la brochure de Charles Braibant, Jacques Meurgey de Tupigny et Yves Metman, Le centenaire de l'atelier des sceaux des Archives nationales, Paris, 1957.

8- Méthode décrite dans Kunstblatt, München, 1846, p. 216

9- Voir les notices des «précurseurs » dans l'ouvrage de Daniel Keller, Le sceau, empreinte de l'Histoire..., op. cit. Voir aussi la notice concernant Fastinger, in NDBA, p. 897-898, par Charles Haudot.

10- Sur Ernest Hauviller, voir NDBA, p. 1459, par Jean-Luc Eichenlaub

11- Kittel, Erich, « Die Siegelsammlungen in den westdeutschen Archiven », in Der Archivar, mai 1964, cahier 2/3, colonnes 225-238

12- Cet inventaire occupe une seule page dans le cahier I de la Landesmünzsammlung. Les sceaux forment la section IV (Abtheilung IV), intitulée « Inventar über die der Landes-Münzsammlung zugewiesenen Abgüsse elsässischer u. lothr. Siegel ». Seuls les premiers envois y sont répertoriés, en 7 séries, pour les années 1883 et 1884 .

13- Kaiserliche Universitäts- und Landesbibliothek, le nom de la BNU à cette époque.

14- Daniel Keller (op. cit.) mentionne les trouvailles faites à son ancien domicile. Ses réalisations sont également bien répandues dans les collections d'archives et de bibliothèques alsaciennes.

15- Achat numéro 73493

16- Avec le dépôt de la famille de Turckheim est entrée une remarquable empreinte en cire rouge du grand sceau de la Ville de Strasbourg, $\mathrm{du} 13^{\mathrm{e}}$ siècle, appendue à une charte de 1591 (MS.Turckheim.033,3, exposée dans les réserves visitables de la BNU).

17- La bibliographie des travaux de Charles Haudot est faite dans le NDBA, p. 1438, par Georges Foessel, et dans l'in memoriam de Daniel Keller, in Revue d'Alsace, 2010.

18- Le site http://www.sigilla.org/ est précisément un projet de ce type, intégrant une part de 3D, mais dont l'Alsace est pour le moment absente. 